\title{
Slicing-Volume: Hybrid 3D/2D Multi-target Selection Technique for Dense Virtual Environments
}

\author{
${ }^{*}$ Roberto A. Montano-Murillo1 †Cuong Nguyen2 Rubaiat Habib Kaziз Sriram Subramanian1 \\ Stephen DiVerdi2 Diego Martinez-Plasencia1
}

1 Interact Lab / Department of Informatics, University of Sussex, Brighton, UK. 2 Adobe Research, San Francisco, California, United States ${ }_{3}$ Adobe Research, Seattle, Washington, United States
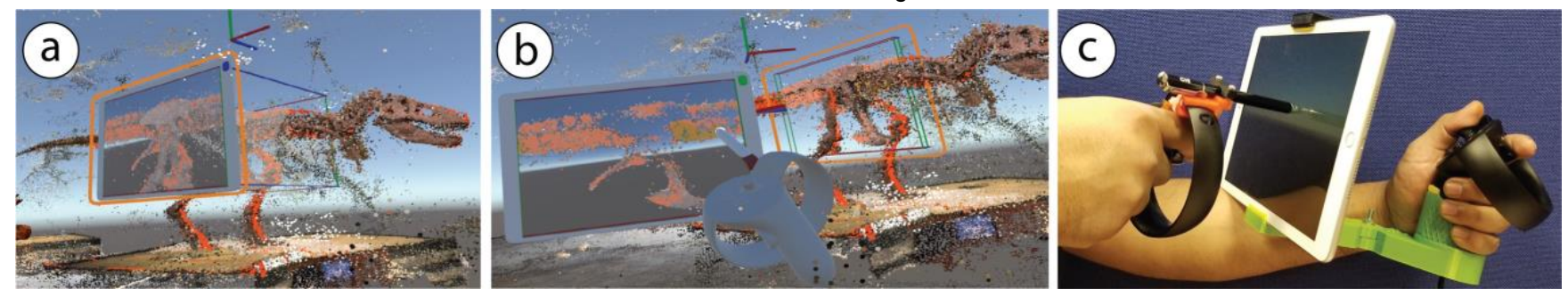

Figure 1: Slicing-Volume is a selection technique for dense VR environments. The user places the volume (which is initially attached to a Virtual Tablet) on the desired area in VR (a), and adjusts the volume size and thickness to explore the model, managing occlusion (b). The points enclosed within the volume are projected to a Virtual Tablet view. Then the user selects the points on the tablet surface using a penand-tablet metaphor. The selection on the tablet is then propagated along the Slicing-Volume thickness and updated in the 3D model. In the real world, the user holds a Real Tablet and a real pen attached to 3D controllers, providing stability and haptic feedback for fine selection (c).

\begin{abstract}
3D selection in dense VR environments (e.g., point clouds) is extremely challenging due to occlusion and imprecise mid-air input modalities (e.g., 3D controllers and hand gestures). In this paper, we propose "Slicing-Volume", a hybrid selection technique that enables simultaneous 3D interaction in mid-air, and a 2D penand-tablet metaphor in VR. Inspired by well-known slicing plane techniques in data visualization, our technique consists of a 3D volume that encloses target objects in mid-air, which are then projected to a $2 \mathrm{D}$ tablet view for precise selection on a tangible physical surface. While slicing techniques and tablets-in-VR have been previously explored, in this paper, we evaluated the potential of this hybrid approach to improve accuracy in highly occluded selection tasks, comparing different multimodal interactions (e.g., Mid-air, Virtual Tablet and Real Tablet). Our results showed that our hybrid technique significantly improved overall accuracy of selection compared to Mid-air selection only, thanks to the added haptic feedback given by the physical tablet surface, rather than the added visualization given by the tablet view.
\end{abstract}

Keywords: 3D selection, hybrid systems, slicing plane, virtual reality, bimanual interaction, tablet.

Index Terms: Computing methodologies $\sim$ Virtual reality.

\section{INTRODUCTION}

Capturing the real-world using 3D scanning and imaging is becoming increasingly popular and widespread across a range of application domains. Examples include photometric scanning [19, 31], medical image analysis (e.g., 3D MRI and CT scans [3]), virtual exploration of infrastructure in VR/AR (e.g., Matterport and ARKit), and VR sculpting \& painting [2, 53]. Target selection plays a critical role for interacting with such unstructured $3 \mathrm{D}$ contents for clean-ups, analysis, and editing. For instance,

\footnotetext{
* R.Montano-Murillo@sussex.ac.uk

† cunguyen@adobe.com
}

scientists analyzing MRI scans often need to use slicing planes to inspect and select relevant areas [30, 34, 42, 49, 51]. However, such selection tasks are challenging due to the complexity of the dense environment [69]. A typical 3D scanned model can contain around six million points. In such a dense space, occlusion is a major challenge for accomplishing selection tasks [39].

Virtual Reality (VR) is a promising platform for 3D data manipulation and analysis $[22,55]$. A key aspect of VR is the high number of degrees of freedom (DoF) used by its input modalities, such as 3D controllers (6-DoF [39]) and mid-air hand gestures (more than 25-DoF [35, 60]). However, despite the freedom of mid-air interactions, these prevalent input modalities lack haptic feedback and stability required for precise interactions [4, 29]. Furthermore, in contrast to 2D interaction (e.g., touchscreens), fine motor control tasks [5, 36] are also physically-demanding in arbitrary 3D scales due to our ergonomic limitations [7].

Recent studies have proposed hybrid techniques that combine the benefits of both free 3D mid-air gestures and precise 2D tactile input $[6,24]$ to aid VR interactions. For example, in SymbiosisSketch [6] having a 2D pen-and-tablet metaphor within a $3 \mathrm{D}$ world, helped in improving precision and ballistic actions by constraining the motion along the $\mathrm{Z}$ axis and providing haptic feedback $[39,40]$. These hybrid approaches have been therefore used for navigation [24, 43], 3D drawing [6] and simple selection (i.e., limited to a low number of targets) [1, 43, 67]. These explorations show promising values of hybrid techniques, particularly to improve the stability of the interaction. However, it is still unclear whether such hybrid approaches could address the occlusion problem for selection tasks in highly dense Virtual Environments (VE's). Specifically, the way these hybrid techniques can be adapted to allow comfortable and accurate selection in dense/highly occluded environments remains unclear.

In this paper, we propose a hybrid selection technique for dense point-clouds in VR. Our technique maps the complex 3D multitarget selection task into a hybrid workflow that combines both mid-air and tablet interactions (see Figure 1). The user is provided with a "Slicing-Volume" (i.e., an extension of slicing planes [30]), that can be freely placed within the VE at highly dense areas, where mid-air selection is difficult (Figurela). The objects within this volume are then presented onto a tablet view, attached to the user's hand, allowing more precise 2D selection (Figure 1b)." 
The mapping from the Slicing-Volume to the tablet reduces the complexity of the selection task. When touching on the tablet, our technique casts a ray from the touch point perpendicular to the tablet surface to select all points along this ray (which length is defined by the Slicing-Volume thickness) (Figure 2c). Thus, users can select 3D points using continuous tactile touch (pen-andtablet metaphor as shown in Figure 1c), which is physically more comfortable and precise [39].

We conducted a user study to explore whether this hybrid technique improves accuracy in dense selection, and if so, which aspects of the system contribute to such improvement (i.e., the stability given by the physical tablet surface, the extra visualization given by the added tablet view, or a combination of both). To answer these questions, we compared three main conditions: (1) Mid-air only, (2) Mid-air \& Virtual Tablet and (3) Mid-air \& Real Tablet in two point-cloud models with different levels of selection difficulty, i.e., occlusion level (highly occluded and mildly occluded).

In summary, unlike current hybrid techniques proposed in the literature (mostly limited to selection with a low number of targets, sparsely distributed across space), the main contributions of our work are:

- We explore the potential of tablet-in-VR approaches to address selection in highly dense/occluded, unstructured VEs.

- We implement a real-time VR selection technique tailored for highly dense and highly occluded environments.

- We conduct a user study to understand the factors influencing users' performance in 3 independent factors: the use of extra visualizations (Mid-air vs Virtual Tablet modes); physical support (Virtual vs Real Tablet modes); and the level of task complexity (highly occluded vs mildly occluded 3D models).

\section{Related Work}

\subsection{Occlusion in 2D/3D Selection}

Object selection has been identified as a fundamental task in 3D user interfaces [39, 46] and one of the primary task in VR interaction [16]. Since 3D object selection is often affected by occlusion (e.g., density, target size, inter-object distance) [4], multiple selection techniques have focused on solutions to overcome this limitation in selection tasks. For instance, Grossman et al. [28] proposed a technique for selection in volumetric displays based on ray intersection (e.g., depth ray, lock ray, smart ray and flower ray) followed by a target disambiguation method consisting on moving a ray-aligned cursor controlled by dimensional device motion. However, the selection in the disambiguation step still needs to be visually confirmed by the user, limiting their applicability to highly occluded environments. Moreover, these techniques are focused to single object where the selection of a big number of targets is not allowed.

Olwal et al. [50] introduced a flexible pointer that bends to avoid obstacles in a single-object selection task on a partially occluded view in a collaborative VE. This technique is highly dependent on the user's point of view limiting thus, the selection of totally occluded objects. Forsberg et al. [25] used a coneshaped volume to select small or distant objects, however, the cone aperture tends to over-select objects around the target.

Progressive refinement techniques have been explored for target disambiguation in locally dense spaces by iteratively splitting the search space into smaller hierarchical regions $[9,38$, 45]. In these techniques, users perform repeated selections until the selection contains a single object. These techniques use several discrete steps to iteratively select an object within a group of interest, which can be tedious to reach the desired target, and are not suitable for highly occluded VEs [38]. Grossman et al. [27] proposed "bubble selection", a 2D area cursor technique that dynamically resizes a circular cursor in order to contain only one object at the time in a $2 \mathrm{D}$ selection task. Further $3 \mathrm{D}$ versions were explored using a 3D volumetric cursor (sphere) instead [20, 56, 65] adding transparency to the volumetric cursor to decrease occlusion over targets. However, these techniques are mostly focused on desktop applications and not suitable for VR. In mobile Augmented Reality (AR), DrillSample [48] is a two-step selection technique that first disambiguates occlusion by showing users an exploded view of all the objects near the selected target. The user can then select the target in the second step. This technique, however, is only applicable to single-object selection.

Clustering methods have been also applied to address the density problem in selection tasks, such as the approach proposed by $\mathrm{Yu}$ et al. [68, 69], where selection tools based on propagation techniques in point-cloud data are used to select group points based on user draws (e.g., using a 2D lasso tool). Similarly, Shan et al. [59] proposed a selection method in dense data sets using 2D circle and polygon tools to select regions of data where a clustering step is based on a voxelization of the selected region to increase clustering accuracy. These are powerful techniques that allow users to select 3D data from 2D inputs however, these techniques are in the frame of data visualization, as they are constrained to PC or touchscreen monitor applications where a single mode gesture interaction for selection is allowed. In contrast, spatial interactions (e.g., hand gestures, controllers, hybrid-interfaces) are relatively less explored.

In VR, numerous techniques have been introduced, taking advantage of the expressive mid-air gestures supported in most VR systems [4]. "Large Scale Cut Plane" [47] and "Yea Big, Yea High" [33] allow the use of mid-air gestures and slicing planes to address occlusion in VR (see [32] for a comparison). Slicing plane is a well-known technique to reduce occlusion and filter data in the scientific visualization community [49]. However, these techniques do not explore additional interaction modes and they were designed mainly for selecting single or partial objects (e.g., a vase or small patches of a 3D mesh), thus they may not be suitable for multi-target selection in dense environments. In contrast, our work explores a more challenging multi-target selection task in a dense VE (containing point clouds), using a technique that maps the $3 \mathrm{D}$ selection task into a hybrid workflow that combines both expressive mid-air and grounded tablet interactions.

\subsection{Selecting with Mobile Devices in Mixed Reality}

\subsubsection{Visualization and Exploration}

A tablet, when tracked in VR, can provide numerous powerful add-ons to how users interact with a VE [13]. Most notably is the use of the tablet screen as an additional tool to support navigation and exploration of the environment. In CAVE systems, Aspin et al. [8] used a tracked tablet and pen to explore complex 3D models. Navigation is a prerequisite of selection in large-scale environment and some techniques combine both navigation and selection on the tablet. For instance, Madeiros et al. [44] mapped finger gestures on tablet to enable selection and manipulation actions. Kim et al. [37] introduced a "finger walking in place" technique for navigation in VR using a tablet touch input. These techniques, however, only let users use $2 \mathrm{D}$ touch gestures to perform these $3 \mathrm{D}$ tasks and do not explore how to integrate them with more expressive mid-air interaction.

Mid-air interaction is arguably the primary mode of interaction in VR. When combined with a 6DOF tracked tablet, most work only explores simple cases such as menu navigation or singleobject selection. For example, Bornik et al. [15], combined 2D pen input on tablet, and mid-air interaction by means of a $3 \mathrm{D}$ controller in VR to enable medical data exploration and manipulation. In AR, Wang and Lindeman [66, 67] employed 3D 


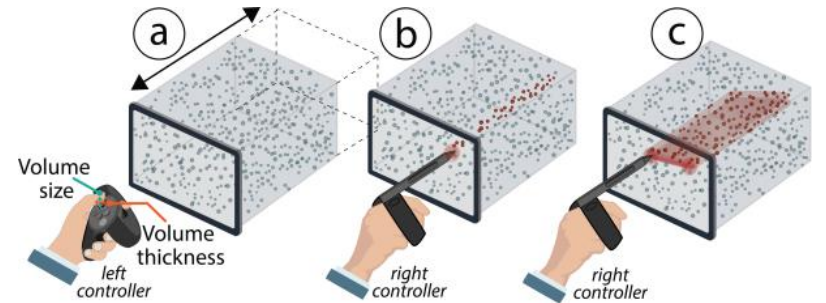

Figure 2: (a) The user increases/decreases the volume size and thickness using the left joystick. (b-c) Our technique casts a ray from the touch point (laser or pen) perpendicular to the tablet surface to select all points along this ray.

controllers for mid-air selection and multitouch tablet input for menu navigation. Reitmayr and Schmalstieg [54] proposed a hybrid AR system for pen and a tablet interaction allowing 2D-3D selection in collaborative tasks. Dias et al. [24] explored hybrid systems and enabled 3D selection by gaze recognition. Finally, Bhaskar-Surale et al. [13] explored the design space for using a multi-touch tablet in solid modelling in VR.

However, these techniques are limited to single or dual object selection and they do not explore the problem of object density. Working at object level, scene knowledge can help selection (snap to plane, object), but this is not suitable for point-clouds. Our paper extends this research by focusing on a more challenging multi-target selection tasks and contributes a selection technique that takes advantage of a seamless hybrid mid-air/tablet workflow to address the density problem in VR selection.

\subsubsection{Haptic Feedback and Stability}

The advantages of mid-air interaction come mainly from the high DOF of the interaction. In VR, with a tracked controller or hand gestures, a user can easily move her hand in mid-air to describe complex shapes and curvature [57]. However, mid-air interaction is known to be imprecise and can cause fatigue over extended use [39]. Prior research has investigated complementing mid-air gestures with physical props to increase stability [17, 18, 41, 52, 58]. Most work, however, focuses only on content creation tasks such as VR sketching [6, 7] and modeling [14, 63]. However, Besancon et all. [12] introduced a hybrid tactile/tangible interaction for $3 \mathrm{D}$ selection, where 2D selection techniques (e.g., lasso tool) are combined with a user-controlled volume extrusion to select in 3D by using a 2D extended tablet-to-screen view. This approach shares similarities with ours, however, it is constrained by the visualization mode (2D views and fixed screen position) while our approach uses an immersive VR approach to support fully exploration of the 3D content in highly occluded scenarios along with a multimodal interaction (mid-air selection, virtual tablet for exploration and a tangible surface to provide stability), none of them explored by [12].

In summary, most of the previously mentioned selection techniques that employ hybrid metaphors (i.e., combine both midair and tablet) do not support fluent multimodal interaction. That is, they constrain the selection to only one modality at a time, either in mid-air or on the physical tablet surface $[1,10,11,26$, 44, 61, 62]. Furthermore, several mid-air techniques in VR/AR only focus on menu navigation or single object selection $[6,13$, $18,66,67]$, not adapting well to highly dense unstructured VEs. In contrast, our approach exploits several modalities, drawing on their strengths to address the requirements of highly dense unstructured VEs. That is, volume placement allows intuitive coarse selection with $6 \mathrm{DoF}$, while tablet interaction allows precise and comfortable selection/refinement. Additionally, we contribute a study that explores the potential factors that increase accuracy in dense multi-target selection when using this hybrid approach, i.e., extra visualization or haptic feedback.

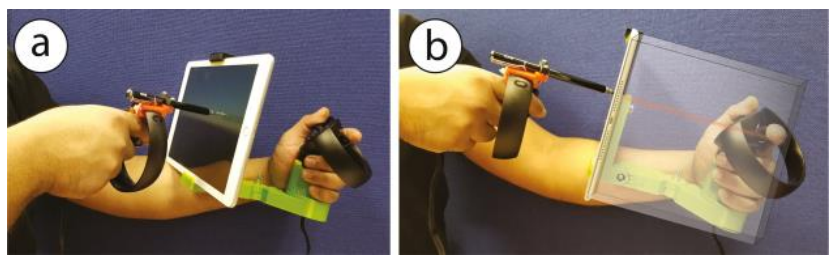

Figure 3: Pen-and-tablet metaphor. Left: A Real Tablet is attached to the left controller using an adjustable 3D-printed holder. A physical stylus is attached to the right controller for touch input. Right: The Slicing-Volume is attached to the tablet in the VE, touch input creates a ray projected along the volume thickness and the points colliding with this ray are then selected.

\section{ImPLEMENTATION AND SETUP}

We implemented our system in Unity (version 2018.4.3), using the point cloud importer "Pcx" [64]. An Oculus Rift and two 3D touch controllers were used for VR interaction. We use an "iPad Air 1" as a physical proxy for our system. The main goal of our system is to explore a hybrid 2D-3D tablet-in- $V R$ approach for improving precision in multi-target selection tasks for dense VEs i.e., leveraging both haptic feedback and exploration offered by typical 2D interaction on a tablet (unlike arbitrary mid-air interaction). To achieve this, our system's key components are: i) Slicing-Volume and ii) 2D-3D mapping (mid-air \& tablet).

\subsection{Slicing Volume}

Inspired by slicing planes commonly used in MRI analysis to explore dense data sets, our Slicing-Volume defines a specific region in the $3 \mathrm{D}$ space to be mapped onto a $2 \mathrm{D}$ tablet view in $\mathrm{VR}$, for multi-object selection tasks [12, 68, 69] (see Figure 1). Contrary to slicing planes that usually visualize the cross-section of the data (the slice), Slicing-Volume uses the orthographic projection view of a virtual camera (adjusting projection size and clipping planes' positions) to capture the content enclosed within its volume, but not the neighboring elements. The rendered view on a Virtual Tablet surface within the VE, allows users to have a visualization tool to filter out elements that surround the desired target selection.

The Slicing-Volume provides a quick and flexible way to specify the initial selection, which can be refined i.e., it can be grabbed, translated and rotated by pressing the grip button on the right controller, to be easily placed and oriented by users during the selection task. Its dimensions can be dynamically adjusted using the joystick on the left controller (see Figure 2a) allowing users to customize the $3 \mathrm{D}$ space mapped on the current Virtual Tablet view e.g., increasing the thickness of volume facilitates the selection of a larger set of points, while decreasing the thickness facilitates precise selection of occluded points (Figure 2). This Slicing-Volume then enables an exploration tool for highly dense data sets, that we employ for more precise selection.

During a selection task, the volume is initially attached to the Virtual Tablet in the VE, which in turn is attached to the left controller position. In the real world, a Real Tablet is attached to the left controller though a 3D-printed holder (see Figure 3), for free translation/rotation using the hand movement. Then, to fix the volume in a position in the $\mathrm{VE}$, the user presses the trigger button on the left controller to decouple the volume from the tablet.

\subsubsection{Selection Modalities}

We designed two different modes to use our Slicing-Volume: (a) combined with the Virtual Tablet only (i.e., providing a visual exploration tool but not haptic feedback) and b) combined with both the Virtual and the Real tablet (i.e., providing both visual exploration and haptic feedback). 
Real Tablet mode: We tracked an iPad air tablet aligned to the virtual one allowing touch interaction on a physical limit using its touchscreen in VR. Selection in this Real Tablet mode enables a pen-and-tablet metaphor, a $10 \mathrm{~cm}$ touch pen was attached to right controller allowing bimanual interaction (see Figure 3).

Virtual Tablet mode: Since this mode does not involve a physical tablet for selection, we employ a virtual laser attached to right controller, using a ray-casting technique to interact with the Virtual Tablet surface allowing also a pen-and-tablet metaphor, but without haptic feedback (Figure 6a).

It is worth mentioning that the physical tablet view is never used for actual visualization (the user is wearing a Head-Mounted Display and immersed in a VE), but only for haptic feedback and touch input. Then, the user always explores the 3D space using the Virtual Tablet view, that can be accompanied by a physical tablet (aligned to the virtual one) or not, depending on the selection modality (see Figure 6).

For selecting elements on the tablet view, the user brushes with the pen/laser on the tablet surface (Virtual or Real) and the strokes are mapped to the Slicing-Volume, i.e., the elements in the tablet view colliding with the laser pen or the real pen are selected and projected along the Slicing-Volume thickness (see Figure 2b-c). Finally, the selection on the tablet is updated in the 3D space.

These two modes (Virtual \& Real) allowed us to independently compare the two primary benefits of tablet-in-VR approaches suggested in the literature (i.e., extra visualization and stability), and thus explore the elements that contribute to improve accuracy of selection (if any). Selection events in both modes are triggered by holding the trigger button on the right controller while brushing on the tablets' surface. Deselection events are allowed and triggered by pressing the " $\mathrm{B}$ " button on the right controller.

\subsection{D-2D Mapping (Mid-air \& Tablet)}

To map 2D strokes from the tablet view to a 3D volumetric space, we first considered the initial size of the Slicing-Volume frontal face to be the same size and aspect ratio as the tablet screen $(15.5 \mathrm{~cm} \times 21 \mathrm{~cm})$, and setting the volume thickness to be equal to the larger tablet side $(21 \mathrm{~cm})$, resulting then $\{15.5 \mathrm{~cm}, 21 \mathrm{~cm}$, $21 \mathrm{~cm}\}$ as our $3 \mathrm{D}$ space unit size $\mathbf{U}(\mathbf{x}, \mathbf{y}, \mathbf{z}) \in \mathbb{R}_{3}$ which is about $6,868 \mathrm{~cm}^{3}$. The volume size $\mathbf{U}$ is then the initial size of the Slicing-Volume in the virtual environment with scale=1. A virtual camera is attached to the frontal face of the Slicing-Volume, this face represents the main perspective to be used for the $2 \mathrm{D}$ tablet view. Changes in the volume size are automatically applied to camera planes per frame, to dynamically adjust the tablet view. Additionally, a coordinate system representation was included to the top right corner of both, tablet and Slicing-Volume to easily match changes in orientation.

We used compute shaders to calculate the number of elements contained within the Slicing-Volume per frame and their interactions with the user's actions (e.g., selection and deselection) with a reduced impact in the framerate. This allows us to handle big data sets using the GPU computation. To facilitate the visual identification of the elements inside the Slicing-Volume in the VE, all of them were highlighted in white (blended the default point color with a pure white).

The position of the elements inside the volume are projected onto the main plane, similar to [12] (i.e., the clipping plane located on the frontal face of the volume) which allows us to quickly detect the collisions from the tablet strokes (by pen/laser selection as shown in Figure 2) and highlight the collided (selected) elements in green (blending the default point color in the point cloud with a pure green) to indicate selection. These highlights (green and white) helps in the visual exploration of the current state of the points that belong and surround the target in the volume from either tablet and VE views.

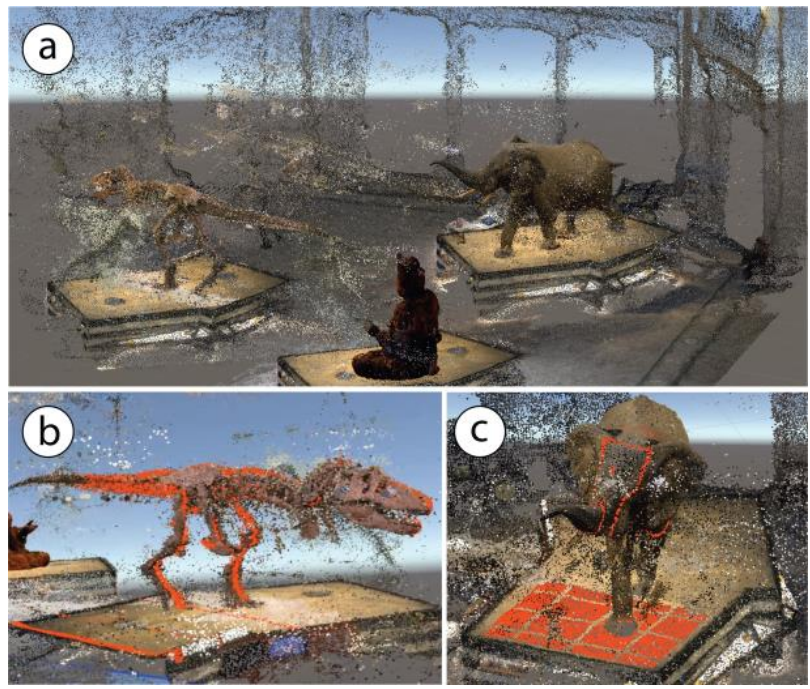

Figure 4: (a) Virtual Environment used in the study ( 1million points). Distribution of target points of the T-Rex (b) and Elephant (c) models i.e., 19,392 points and 7,293 points respectively.

\section{USER STUdY}

We conducted a within-subjects user study in VR to test our system. Our study aimed to (1) explore whether our approach improves user performance in selection tasks and (2) gain insight about the cause of the potential benefits obtained (i.e., visualization vs haptic feedback). We asked participants to select a set of target points from a dense point-cloud in VR (Figure 4).

As we are interested in understanding the benefits of having a tangible tablet for this task in VR, we compared our two selection modalities (see Figure 6): "pen selection" using both Virtual and Real tablets (i.e., involving Slicing-Volume exploration tool and haptic feedback) and "laser selection" using the Virtual Tablet only (i.e., involving Slicing-Volume exploration tool but not haptic feedback). A baseline condition was also included in the study, consisting of a standard 6-DOF brushing-based selection technique i.e., Mid-air only selection using one 3D controller without the use of Slicing-Volume tool nor tablet modalities. The detailed design of these conditions is described in the Experimental Conditions section and depicted in Figure 5.

\subsection{Participants}

Twelve participants ( 2 females, mean age $=28.08$ years old, $\mathrm{SD}=4.62$ ) took part in our study. They were all right-handed with no previous experience on VR. The recruitment criteria only included having normal or corrected-to-normal vision and having no injuries on their hands and/or arms. The local ethics committee approved this study. A full session took about $60 \mathrm{~min}$ and participants were not paid for their participation.

\subsection{Task Point-Cloud Model}

We designed a dense point cloud environment for the study. Our point cloud model consists of 984,613 points ( $\sim 1$ million). The model shows a museum with three sculptures (i.e., a statue, a TRex and an Elephant, Figure 4). The statue was used for training. Then, the T-Rex and Elephant were used for the main tasks. Our task design was similar to [12].

Each structure had a set of target points (i.e. users had to select them) highlighted in orange. Target points were distributed in geometric patterns, and each sculpture represented a different level of difficulty, defined in terms of occlusion level. We defined the occlusion level by the point density per space unit $(\mathbf{U})$. The Elephant model has an average density of 22,597 points/U (level 


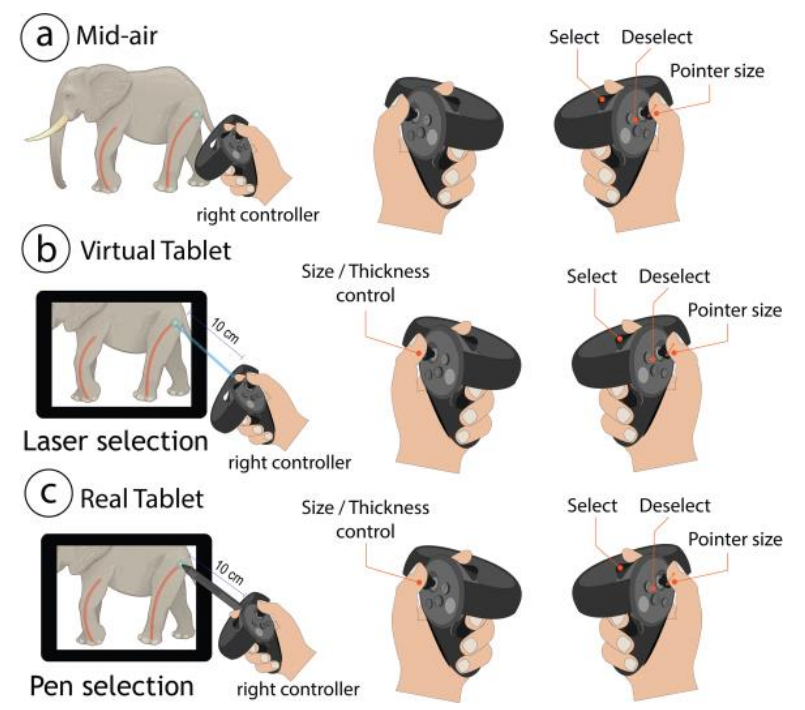

Figure 5: Controller actions for the three interaction techniques.

of occlusion), containing 7,293 target points (Figure 4c). Then, the T-Rex model has an average density of 42,687 points/ $\mathbf{U}$, containing 19,392 target points (Figure 4b). This means that the density of the T-Rex model was almost two times more occluded and therefore more challenging than the elephant model.

The rationale behind our scenario and manual task design lays on two main factors. First, the manual selection of target points allowed us to make clear the difference in selection difficulty in terms of occlusion between tasks. That is, the target points in the T-Rex model is in a much denser region and is more difficult than the Elephant model (as shown in Figure 4b-c). Second, we distribute the target points such that they do not cover a specific body parts of the model (e.g., one leg or the head).

While this decision makes our tasks less realistic, it helps us ensure the tasks are equally challenging toward all of the conditions. In a pilot testing, we found that if the target points are specific (e.g., we placed the target points covering only the $\mathrm{T}$ Rex's head), the Slicing-Volume technique can capture all of the targets in a short time with high accuracy. This does not fit our study goal, i.e., the aim of our design was (1) make a fair comparison between all the conditions and (2) explore when our hybrid system could be more useful (i.e., for highly/mildly occluded models), being the task challenge clearly distinct.

\subsection{Experimental Conditions}

We designed our three experimental conditions to be as similar as possible so that measured effects are exclusively due to the interaction technique (Mid-air, Virtual Tablet and Real Tablet) instead of other parameters. In all the conditions, the main actions (select, deselect, and pointer size adjustment) were mapped to the right controller. In the two conditions involving Slicing-Volume tool, the left controller was used to adjust the volume dimensions (see Figure 5). In these two conditions, users were allowed to freely transition between selection modalities (Mid-air and Slicing-Volume selection) to complete the task, enabling users to select the targets by combining the benefit of each selection mode. Each participant selected the target points in the two models (Elephant and T-Rex) using the three conditions.

Condition 1 (Mid-air only): This is our baseline condition. Participants were given only the right controller and asked to select the target points by brushing in mid-air. A floating sphere in front of the controller indicated the pointer to select/deselect. Points colliding with this sphere are then selected/deselected. The

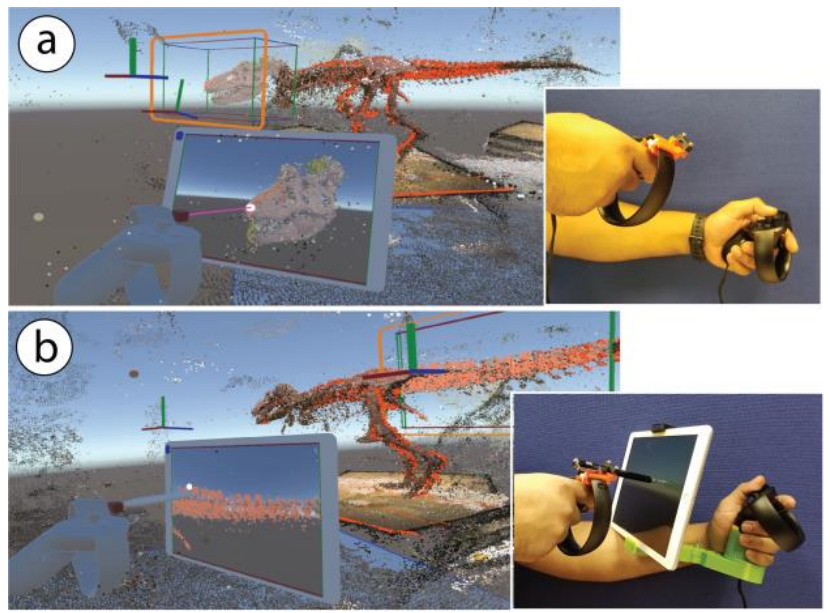

Figure 6: (a) Laser selection: Participants select on the tablet view using a visible "laser" beam $(10 \mathrm{~cm})$ with no tangible feedback. (b) Pen selection: Participants select using an actual pen $(10 \mathrm{~cm}$ long) on the tangible tablet surface (the pen is virtually represented).

size of the pointer could be increased/decreased (with a minimum diameter of $0.7 \mathrm{~cm}$ ) to support varying levels of precision. Neither Slicing-Volume tool nor tablets were used in this condition.

Condition 2 (Virtual Tablet): Participants were given two controllers and, in addition to the mid-air selection, they were also allowed to use the Slicing-Volume tool. They could place the volume (which was initially attached to the Virtual Tablet representation) in the desired area and adjust its size and thickness to explore the models. The points enclosed within the volume were mapped to the Virtual Tablet view where participants could select/deselect the target points by "laser selection" modality (see Figure 6a). Selection/deselection events were enabled by the "laser" tip $(0.7 \mathrm{~cm}$ diameter) colliding with the Virtual Tablet surface. The selection in the Virtual Tablet was then propagated along the Slicing-Volume thickness and updated in the 3D model.

Condition 3 (Real Tablet): In addition to Mid-air selection and Virtual Tablet representation with Slicing-Volume tool, participants were given an iPad air tablet which was attached to the left controller through an adjustable 3D-printed holder (see Figure 6b). A physical pen was then attached to the right controller (replacing "laser selection"). Selection/deselection events were enabled by the pen tip $(0.7 \mathrm{~cm}$ diameter $)$ touching the Real Tablet touchscreen (see Figure 6b). The selection in the Real Tablet was then propagated along the Slicing-Volume thickness and updated in the 3D model.

\subsection{Experimental Setup}

Participants were asked to sit next to a semicircle-shaped table and adjust the chair height for comfort. They then wore an Oculus Rift headset (see Figure 7). Participants could rest the Real Tablet on the table to avoid fatigue. Participants could also translate/rotate the whole environment to interact with it while sitting (avoiding overstretching), these spatial transformations were carried out by manipulating a blue cube placed under the model. Cube transformations (enabled by pressing the grip button on the right controller when inside this cube) were mapped to the main model. Subsequently, participants performed a training stage that lasted 15 minutes where they were allowed get familiar with the system and practice the selection task (targets on the statue model only). After the training, we presented the two models ( $\mathrm{T}$ Rex and Elephant) per condition (6 trials per participant) with their target points highlighted in orange. The order of the conditions (Mid-air only, Virtual Tablet and Real Tablet) as well 


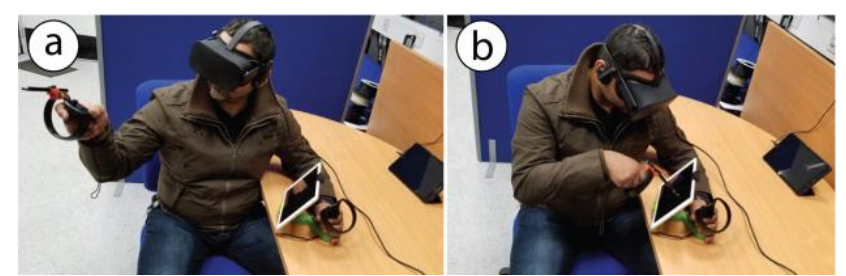

Figure 7: Experimental setup.

as the order of the models were counterbalanced across participants. In each trial, users selected the orange target points and confirmed their selection by placing the right controller in a designated red area and pressing the trigger button (when they considered selection was finished), allowing them to progress to the next trial. Each trial was limited to 10 minutes in duration to avoid fatigue (determined based on a prior pilot study). That is, when 10 minutes elapsed from the beginning of the trial, they were forced to finish the current trial and continue the next one.

\subsection{Variables Measured}

We measured the selection accuracy using on two strategies: F1 score and Matthews correlation coefficient (MCC) [12, 68, 69]. Both, F1 and MCC scores were computed from TP (true positive: number of target points correctly selected), FP (false positive: number of points incorrectly selected), and FN (false negative: number of target points that were not selected). Additionally MCC also considered TN (true negatives: number of points correctly non-selected). F1 score is a weighted average of precision $\mathrm{P}=$ $\mathrm{TP} /(\mathrm{TP}+\mathrm{FP})$ and recall $\mathrm{R}=\mathrm{TP} /(\mathrm{TP}+\mathrm{FN})$. Then, $\mathrm{F} 1$ is computed as $\mathrm{F} 1=2 \cdot(\mathrm{P} \cdot \mathrm{R}) /(\mathrm{P}+\mathrm{R})$. While MCC score is computed as:

$\mathrm{MCC}=\frac{(T P * T N-F P * F N)}{\sqrt{((T P * F P)(T P * F N)(T N * F P)(T N * F N))}}$

A F1 score of 1 indicates perfect selection performance and 0 otherwise. Similarly, a MCC score of 1 suggests a perfect performance while -1 the worst one. As shown in Figure 8, the F1 and MCC scores highly correlates for all conditions evaluated in the experiment. We therefore use only F1 scores to present the results in terms of accuracy in our analysis (significance, and paired tests are always in agreement, using either metric). Then, when we employ the terms Over-Selection and Under-Selection in our analysis, refer to FP and FN respectively.

Time measures were also recorded, we measured time of selection (time that participants took in selection mode, while pressing trigger), time of deselection (time that participants took in deselection mode while pressing "B") and total time which represents the Task Completion Time (TCT) in each model i.e., including the time participants took: (a) in selection mode, (b) in deselection mode, (c) observation (no mode activated) and (d) manipulating the volume. Additionally, we assessed participants' load index in each condition using a raw TLX scale.

Since we are comparing experimental conditions (tablet modes) with controls (our baseline Mid-air only mode) we employed Standard Error of Mean (SEM) to represent error bars in our plots according to Rule 4 in [23].

\section{Results}

We conducted One-way Repeated Measures ANOVA tests in our analyses supported by Shapiro-Wilk Normality Tests in the three conditions as well as a Normal Q-Q plots and Mauchly's test of sphericity (i.e., sphericity not rejected) to ensure that the normal distribution and homogeneity of the variance assumptions were not violated. Partial eta squared $\left(\eta^{2}\right)$ is reported as a measure of effect size, according to Cohen [21].
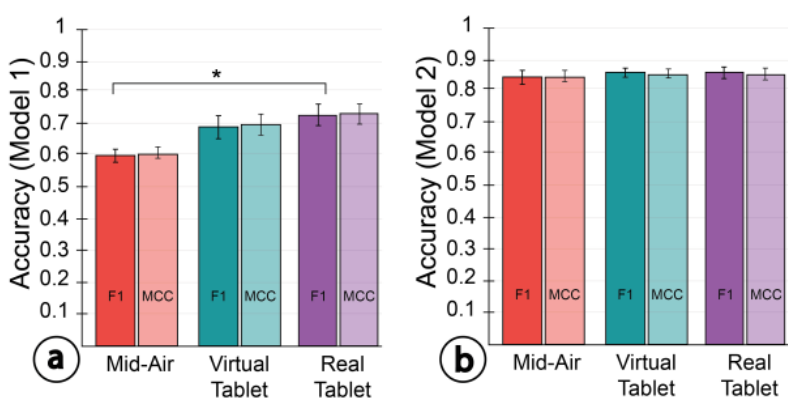

Figure 8: Results on Accuracy (F1\&MCC) for Model 1 (left) and Model 2 (right). Error bars represent SEM. ${ }^{*}=p<0.05$.

\subsection{Accuracy}

A One-way Repeated Measures ANOVA was conducted to compare the effect of the interaction technique (i.e., Mid-air only, Virtual Tablet and Real Tablet) on participants' accuracy of selection in Model 1 (T-Rex). Results showed significant effect of the interaction technique $\left(\mathrm{F}_{(2,22)}=3.27, \quad p=0.017, \eta^{2}=0.557\right)$. Comparison tests with Bonferroni correction showed a significant difference between Mid-air and Real Tablet conditions $(p=0.039)$ but we found no difference between Mid-air and Virtual Tablet conditions $(p=0.135)$. See Figure $8 \mathrm{a}$, for mean F1\&MCC scores.

The data collected from Model 2 (Elephant) did not meet the homogeneity of the variance assumption $(\mathrm{p}=0.002)$, and we therefore carried out a non-parametric One-Way Repeated Measures ANOVA (Related-Samples Friedman's Two-Way Analysis of Variance by Ranks). Results showed no significant effect of the interaction technique on participants accuracy of selection $(\mathrm{p}=0.92)$. See Figure $8 b$, for mean F1\&MCC scores.

\subsection{Over-Selection and Under-Selection}

A One-way Repeated Measures ANOVA was then conducted to compare the effect of the three interaction techniques on participants' Over-Selection and Under-Selection in Model1 (TRex). Results showed significant effect of the interaction technique on Over-Selection $\left(\mathrm{F}_{(2,22)}=4.04, p=0.05, \eta^{2}=0.447\right)$. Comparison test with Bonferroni correction showed significant difference only between Mid-air and Real Tablet conditions $(p=0.04)$. Results also showed a significant effect of the interaction technique on participants' Under-Selection in Model $1\left(\mathrm{~F}_{(2,20)}=4.04, p<0.001, \eta^{2}=0.817\right)$. Comparison tests showed a significant difference between both Mid-air only and Virtual Tablet conditions $(p<0.001)$ and between Mid-air only and Real Tablet conditions ( $p=0.016$ ) (see Figure $9 a$ ).

The same test was conducted for Model 2 (Elephant). However, no significant effect was found of the interaction technique on Over-Selection $\left(\mathrm{F}_{(2,22)}=0.546, p=0.596, \eta^{2}=0.098\right)$ nor UnderSelection $\left(\mathrm{F}_{(2,22)}=0.460, p=0.644, \eta^{2}=0.084\right)$, (Figure $\left.9 b\right)$.

\subsection{Time}

A One-way Repeated Measures ANOVA was again conducted, to compare now the effect of the interaction technique on participants' time of selection, time of deselection and total time in Model 1 (T-Rex). Results showed significant effect only for time of selection $\left(\mathrm{F}_{(2,22)}=18.47, p<0.001, \eta^{2}=0.787\right)$. Comparison tests with Bonferroni correction showed a significant difference between both Mid-air only and Virtual Tablet conditions $(p=0.001)$ and between Mid-air only and Real Tablet conditions $(p=0.01)$, (see Figure $9 \mathrm{c})$.

The same test was conducted for Model 2 (Elephant). Significant effect of the interaction technique on total time was found $\left(\mathrm{F}(2,22)=12.29, p<0.002, \eta^{2}=0.711\right)$. Comparison tests with 

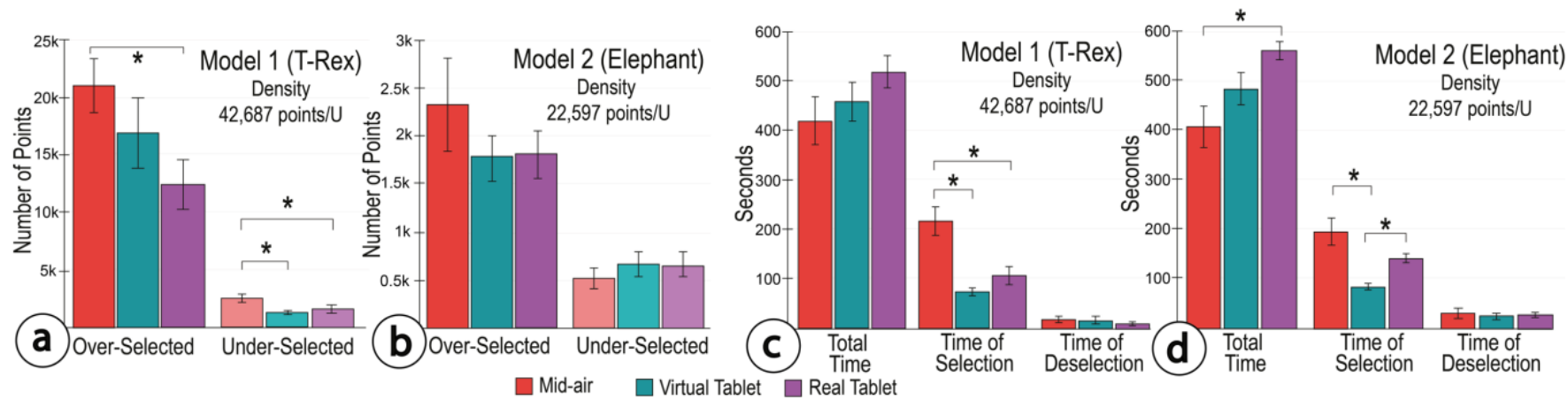

Figure 9: Results on Over-Selection (FP: false positive) and Under-Selection (FN: false negative) for Model 1 (a) and Model 2 (b). Results on Time in Model 1 (c) and Model 2 (d). Error bars represent SEM. ${ }^{*}=p<0.05$

Bonferroni correction showed a significant difference only between Mid-air only and Real Tablet conditions $(p=0.002)$. Significant effect was also observed for time of selection $\left(\mathrm{F}_{(2,22)}=12.29, \quad p<0.002, \quad \eta^{2}=0.711\right)$. Comparison tests with Bonferroni correction showed a significant difference between both Mid-air only and Virtual Tablet conditions $(p=0.003)$ and between Virtual Tablet and Real Tablet conditions $(p<0.001)$.

However, no significant effect of the interaction technique on deselection time was observed for Model $2\left(\mathrm{~F}_{(2,22)}=0.15, p<0.85\right.$, $\eta^{2}=0.030$ ), (see Figure 9d).

\subsection{Load Index}

We also analyzed participants' self-reported load index using a raw TLX scale. A One-way Repeated Measures ANOVA was conducted to compare the effect of the interaction technique on participants' load index, (specifically on: mental demand, physical demand, temporal demand, performance, effort and frustration) in both Models. Results showed significant effect of the interaction technique only on performance $\left(\mathrm{F}_{(2,22)}=3.99\right.$, $\left.p=0.05, \eta^{2}=0.444\right)$. Comparison test with Bonferroni corrections showed a significant difference only between Mid-air Only and Real Tablet conditions ( $p=0.04$ ). See Figure 10 for mean scores.

\section{Discussion}

The results from our user evaluation show that our hybrid interaction technique actually improved participants' accuracy of selection in a highly occluded task environment. However, we found that the participants' accuracy depended on the occlusion level of the task. Recall that our two models differ in terms of occlusion: Model 1 T-Rex (42,687 points/U) is more occluded than Model 2 elephant $(22,597$ points/U). In Model 2, the difference in participants' accuracy is minimal in both F1 and

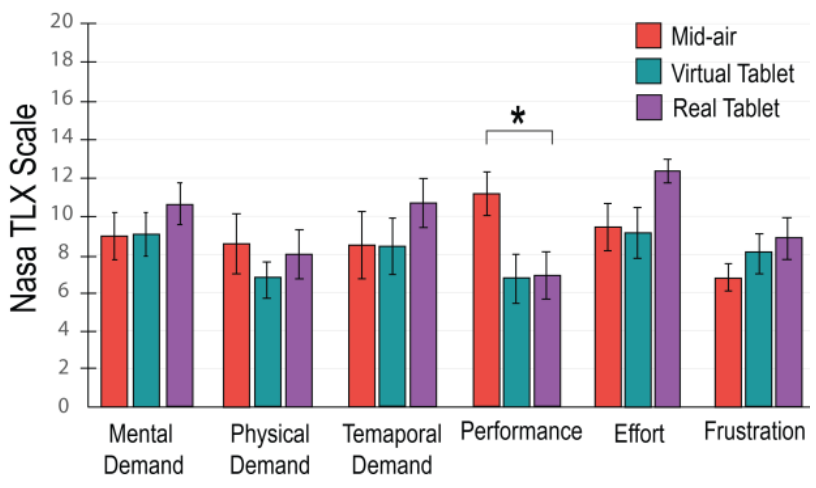

Figure 10: Results on subjective load index in both models for each interaction technique. Low values represent a low negative impact over each factor tested (e.g., performance values, $0=$ perfect $\& 20=$ total failure) Error bars represent SEM. ${ }^{*}=p<0.05$.
MCC scores, suggesting that the hybrid workflow may not be as advantageous for improving the selection performance in this particular scenario (see Figure 8b).

However, in Model 1, we found that participants in the Real Tablet condition were significantly more accurate than in the Midair condition. This finding suggests that the reduction of multitarget selection into a hybrid workflow (that combines mid-air gestures, 2D selection, and physical proxies) through using a Slicing-Volume was more beneficial than the baseline Mid-air only condition during the selection task. Although the Virtual Tablet condition also outperforms the Mid-air condition, the difference was not found to be statistically significant. This result suggests that having only the additional visualization tool (Virtual Tablet view) may not be enough to significantly improve accuracy compared with Mid-air condition in a highly occluded environment. This suggests that the physical surface of the Real Tablet is clearly beneficial due to the extra haptic and stability support. Furthermore, these results still hold when considering Over-Selection and Under- Selection measures of Model 1.

Under-Selection refers to target points participants missed during the task. When a scene is highly occluded, it affects a user's ability to locate the target [4]. Thus, participants trying to select in an occluded environment may suffer more from UnderSelection. In our study, during both Virtual and Real Tablet conditions, participants significantly under-selected less compared with the Mid-air only condition. This suggests that participants benefited from the hybrid workflow, which used the SlicingVolume visualization to help disambiguate occluded targets. However, our results showed no benefit of the tablet conditions (Virtual and Real) when selecting Model 2. In summary, in terms of Under-Selection, having an extra visualization (given by the Slicing-Volume tool) helped to improve participants' performance, rather than having haptic feedback (given by the physical tablet surface) for the most occluded model only.

Over-Selection refers to points participants unintentionally selected. This measure reflects the participants' ability to trace the target points to make the selection. As shown in our results, participants in the Mid-air only condition made much more OverSelection than in the other two conditions, supporting that mid-air gestures are known to be inaccurate [7]. However, we found that only during the Real Tablet condition participants over-selected

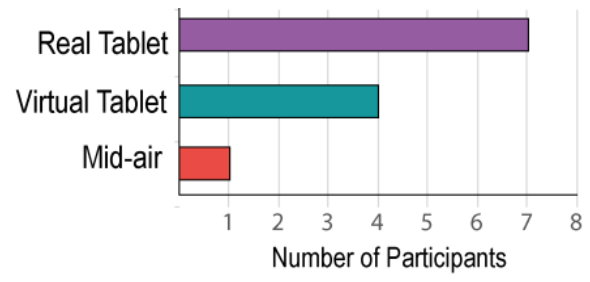

Figure 11: Participants' preferred interaction technique. 
significantly less than during the Mid-air only condition. This suggests that participants benefited from the physical tablet surface thanks to its stability and the selected more accurately.

However, again our results showed no benefit of the tablet conditions (Virtual and Real) when selecting Model 2. In summary, in terms of Over-Selection (in a highly occluded environment), having haptic (given by the physical tablet surface) helped to improve participants' performance, rather than having an extra visualization (given by the Slicing-Volume tool) for the most occluded model only.

These findings further show that our technique complements well the inaccuracy of mid-air gestures and can act as an effective tool to inspect/select target points in highly occluded VE.

It is worth mentioning that, the Real Tablet comes with extra weight, which may have affected participants' ability to quickly execute actions in the study. For instance, in the measured time (Figure $9 \mathrm{c}-\mathrm{d}$ ), we found that participants spent more total time in the Real Tablet condition. This could be due to the extra maneuvers they had to execute to look at the tablet and adjust the volume thickness. However, total time was only significantly higher when selecting Model 2 (involving lower points density). We interpret these results as our hybrid system is more effective for higher dense models (e.g., our Model 1) i.e., improving accuracy without significantly increasing the TCT.

One interesting observation in our results is that overall participants took more time of selection (i.e., in "selection mode" while pressing trigger) in the Mid-air only condition while selecting both models (Figure 9c-d). That is, even when participants took more total time (i.e., TCT) during the tablet conditions, they spent significantly more time in "selection mode" during the Mid-air only condition. We interpret these results as participants could select target points faster and felt more confident about their selection (i.e., spending less time selecting) due to both stability and extra visualization provided by the Virtual and Real tablets.

Regarding participants' subjective load index collected using a raw TLX scale, we found no effect of the interaction techniques on mental demand, physical demand, temporal demand, effort and frustration. Although we observed trend showing higher scores for the Real Tablet condition, it was found non-significant. However, we found that participants perceived significantly better performance when using the Real Tablet (Figure 10).

There are certain trade-offs in the design of the Real Tablet that may affect the user experience. For instance, the physical sensation of using the Real Tablet helped participants feel more grounded during the task. When asked about the preferred interaction technique, the majority of the participants preferred the Real Tablet (see Figure 11). For example, P5 commented "the feeling of having a physical limit to select makes it more real", P7 mentioned "a physical surface in selection is useful" and "the Virtual Tablet feels less natural than the Real Tablet". P8 said "the Virtual Tablet allows me to select faster, but I may tend to overselect" and "the Real Tablet gives the tactile cues to link with the real world, that feels cool".

Overall, our hybrid approach showed positive results suggesting relevant benefits to be used in highly occluded VR models (e.g., editing: point-cloud models or dense 3D painting). The reduction of the multi-target selection into the tablet space through the Slicing-Volume tool enable users to manage occlusion and achieved better selection performance. It also complements the imprecise nature of mid-air selection, allowing users to select with higher selection accuracy. While hybrid approaches have been previously explored in the literature and suggested to provide advantages in VR tasks, in this paper we provide an analysis of the benefits of this novel hybrid workflow in a dense VR selection task with different levels of density and occlusion.
Whit our results, we expect to contribute insights to consider when designing tablet-in-VR approaches, particularly those involving dense environments

\section{Limitations AND FUTURE WORK}

One possible limitation of our method is that participants manually placed the Slicing-Volume in the desired area, instead of using an object placement technique. However, we see benefits of our decision in two main ways. First, to allow users full control over the Slicing-Volume transformations (translation, scaling and rotation) based on their needs e.g., fixing the Slicing-Volume position according to the desired view of the workspace/targets (this is according to [12]). Second, to support a structureindependent selection technique. Usually object placement techniques may depend on pre-processed datasets, constraining the applicability of the technique to such structure-dependent data (this is according to [12]). Since our manual volume placement is structure-independent our selection can support both dataset types while allowing users full control over the Slicing-Volume.

Additionally, while the use of a manual Slicing-Volume placement can be dependent of users' skills for manipulation, the training time becomes important to better exploit the potential of the full control over the placement and transformations. Then, a further study is needed to compare the advantages of manual placement control vs previously explored object placement techniques, and also explore possible integrations between our Slicing-Volume and object placement techniques.

Finally, for experimental purposes we kept all conditions simple with only few tools enabled. However, for future work we will add more tools to our system such as lasso, undo, delete, and save. Another limitation of our study is that it was done with only a small set of novice VR users. Given the positive results from this preliminary analysis, a direction for future work is to evaluate our system with professional 3D designers to gain deeper insight of the benefits of our hybrid system in more application scenarios such as actual editing e.g., point cloud, dense painting and sculpting tasks.

\section{CONCLUSION}

We presented a novel hybrid interaction technique called SlicingVolume for selection in highly occluded point cloud models in VR. Our approach provides users with a 3D volume visualization that can enclose target objects in mid-air. Users can use this volume to inspect and filter out potential target points for selection. These points are then mapped to a $2 \mathrm{D}$ tactile tablet surface so that users can select with haptic feedback given by the tablet surface. We tested our system in a user study and found that our interaction technique improved the accuracy of selection in a highly occluded point cloud model in VR. Our results also provide insights into the specific benefits of our technique. We found that the mapping of the selection region into a $2 \mathrm{D}$ tactile surface on the tablet is generally the most beneficial when it comes to aiding the selection. The additional volume visualization is also favourable to help users filter occluded points, but only when used together with the tactile tablet surface. Overall, these findings show that our technique can act as an effective tool to help users inspect and select target points in dense VE.

\section{ACKNOWLEDGEMENTS}

This work was partly supported by the ERC Advanced Grant (number 787413) and the Royal Academy of Engineering through their Chairs in Emerging Technology Program. 


\section{References}

[1] L. Afonso, P. Dias, C. Ferreira, and B. S. Santos, "Effect of handavatar in a selection task using a tablet as input device in an immersive virtual environment." IEEE Symposium on $3 D$ User Interfaces (3DUI) pp. 247-248, 2017.

[2] M. Agrawala, A. C. Beers, and M. Levoy, "3D painting on scanned surfaces." Proceedings of the 1995 symposium on Interactive 3D graphics pp. 145-ff., 1995.

[3] F. Altahawi, and N. Subhas, "3D MRI in Musculoskeletal Imaging: Current and Future Applications," Current Radiology Reports, vol. 6 , no. 8, pp. 27, 2018.

[4] F. Argelaguet, and C. Andujar, "A survey of 3D object selection techniques for virtual environments," Computers \& Graphics, vol. 37, no. 3, pp. 121-136, 2013

[5] R. Arora, I. Darolia, V. P. Namboodiri, K. Singh, and A. Bousseau, "SketchSoup: Exploratory Ideation Using Design Sketches." Computer Graphics Forum pp. 302-312, 2017.

[6] R. Arora, R. Habib Kazi, T. Grossman, G. Fitzmaurice, and K. Singh, "SymbiosisSketch: Combining 2D \& 3D Sketching for Designing Detailed 3D Objects in Situ." Proceedings of the 2018 CHI Conference on Human Factors in Computing Systems pp. 185, 2018.

[7] R. Arora, R. H. Kazi, F. Anderson, T. Grossman, K. Singh, and G. W. Fitzmaurice, "Experimental Evaluation of Sketching on Surfaces in VR." CHI pp. 5643-5654, 2017.

[8] R. Aspin, and K. H. Le, "Augmenting the CAVE: An initial study into close focused, inward looking, exploration in IPT systems." 11th IEEE International Symposium on Distributed Simulation and RealTime Applications (DS-RT'07) pp. 217-224, 2007.

[9] F. Bacim, R. Kopper, and D. A. Bowman, "Design and evaluation of 3D selection techniques based on progressive refinement," International Journal of Human-Computer Studies, vol. 71, no. 7-8, pp. 785-802, 2013.

[10] H. Benko, and S. Feiner, "Balloon selection: A multi-finger technique for accurate low-fatigue 3d selection." IEEE Symposium on 3D User Interfaces pp., 2007.

[11] L. Besançon, P. Issartel, M. Ammi, and T. Isenberg, "Hybrid tactile/tangible interaction for 3D data exploration," IEEE transactions on visualization and computer graphics, vol. 23, no. 1, pp. 881-890, 2017.

[12] L. Besançon, M. Sereno, L. Yu, M. Ammi, and T. Isenberg, "Hybrid Touch/Tangible Spatial 3D Data Selection." Computer Graphics Forum pp. 553-567, 2019.

[13] H. Bhaskar Surale, A. Gupta, M. Hancock, and D. Vogel, "TabletInVR: Exploring the Design Space for Using a Multi-Touch Tablet in Virtual Reality," in Proceedings of the 2019 CHI Conference on Human Factors in Computing Systems, Glasgow, Scotland 2019.

[14] M. Billinghurst, S. Baldis, L. Matheson, and M. Philips, "3D palette: a virtual reality content creation tool." Proceedings of the ACM symposium on Virtual reality software and technology pp. 155-156, 1997.

[15] A. Bornik, R. Beichel, E. Kruijff, B. Reitinger, and D. Schmalstieg, "A hybrid user interface for manipulation of volumetric medical data." null pp. 29-36, 2006.

[16] D. A. Bowman, and L. F. Hodges, "Formalizing the design, evaluation, and application of interaction techniques for immersive virtual environments," Journal of Visual Languages \& Computing, vol. 10, no. 1, pp. 37-53, 1999.

[17] D. A. Bowman, M. Setareh, M. S. Pinho, N. Ali, A. Kalita, Y. Lee, J. Lucas, M. Gracey, M. Kothapalli, and Q. Zhu, "Virtual-SAP: an immersive tool for visualizing the response of building structures to environmental conditions." IEEE Virtual Reality Proceedings pp. 243-250, 2003.

[18] D. A. Bowman, and C. A. Wingrave, "Design and evaluation of menu systems for immersive virtual environments." Proceedings IEEE Virtual Reality 2001 pp. 149-156, 2001.

[19] A. Cheveau, "3D Scanning Solution for Textured Object using Photometric Stereo with Multiple Known Light Sources." Archiving Conference pp. 6-9, 2018.
[20] A. Cockburn, and A. Firth, "Improving the acquisition of small targets," People and Computers XVII-Designing for Society, pp. 181-196: Springer, 2004.

[21] J. Cohen, "Statistical power analysis for the behavioral sciences . Hilsdale," NJ: Lawrence Earlbaum Associates, vol. 2, 1988.

[22] C. Cruz-Neira, J. Leigh, M. Papka, C. Barnes, S. M. Cohen, S. Das, R. Engelmann, R. Hudson, T. Roy, and L. Siegel, "Scientists in wonderland: A report on visualization applications in the CAVE virtual reality environment." Proceedings of 1993 IEEE Research Properties in Virtual Reality Symposium pp. 59-66, 1993.

[23] G. Cumming, F. Fidler, and D. L. Vaux, "Error bars in experimental biology," The Journal of cell biology, vol. 177, no. 1, pp. 7-11, 2007.

[24] P. Dias, L. Afonso, S. Eliseu, and B. S. Santos, "Mobile devices for interaction in immersive virtual environments." Proceedings of the 2018 International Conference on Advanced Visual Interfaces pp. $19,2018$.

[25] A. Forsberg, K. Herndon, and R. Zeleznik, "Aperture based selection for immersive virtual environments." ACM Symposium on User Interface Software and Technology pp. 95-96, 1996.

[26] A. Giesler, D. Valkov, and K. Hinrichs, "Void shadows: multi-touch interaction with stereoscopic objects on the tabletop." Proceedings of the 2nd ACM symposium on Spatial user interaction pp. 104-112, 2014.

[27] T. Grossman, and R. Balakrishnan, "The bubble cursor: enhancing target acquisition by dynamic resizing of the cursor's activation area." Proceedings of the SIGCHI conference on Human factors in computing systems pp. 281-290, 2005.

[28] T. Grossman, and R. Balakrishnan, "The design and evaluation of selection techniques for 3D volumetric displays." Proceedings of the 19th annual ACM symposium on User interface software and technology pp. 3-12, 2006.

[29] K. P. Herndon, A. van Dam, and M. Gleicher, "The challenges of 3D interaction: a CHI'94 workshop," ACM SIGCHI Bulletin, vol. 26, no. 4, pp. 36-43, 1994.

[30] K. Hinckley, R. Pausch, J. C. Goble, and N. F. Kassell, "Passive real-world interface props for neurosurgical visualization," in Proceedings of the SIGCHI Conference on Human Factors in Computing Systems, Boston, Massachusetts, USA, pp. 452-458 1994.

[31] S. Izadi, D. Kim, O. Hilliges, D. Molyneaux, R. Newcombe, P. Kohli, J. Shotton, S. Hodges, D. Freeman, and A. Davison, "KinectFusion: real-time $3 \mathrm{D}$ reconstruction and interaction using a moving depth camera." Proceedings of the 24th annual ACM symposium on User interface software and technology pp. 559-568, 2011.

[32] B. Jackson, K. Beckham, A. K. Cohen, and B. C. Heggeseth, "Comparing Convex Region-of-Interest Selection Techniques for Surface Geometry." 25th ACM Symposium on Virtual Reality Software and Technology pp. 34, 2019.

[33] B. Jackson, B. Jelke, and G. Brown, "Yea Big, Yea High: A 3D User Interface for Surface Selection by Progressive Refinement in Virtual Environments." IEEE Conference on Virtual Reality and $3 D$ User Interfaces (VR) pp. 320-326, 2018.

[34] F. Janoos, B. Nouanesengsy, R. Machiraju, H. W. Shen, S. Sammet, M. Knopp, and I. Á. Mórocz, "Visual analysis of brain activity from fMRI data." Computer Graphics Forum pp. 903-910, 2009.

[35] L. A. Jones, and S. J. Lederman, Human hand function: Oxford University Press, 2006.

[36] D. F. Keefe, R. C. Zeleznik, and D. H. Laidlaw, "Drawing on air: Input techniques for controlled 3D line illustration," IEEE Transactions on Visualization and Computer Graphics, vol. 13, no. 5, pp. 1067-1081, 2007.

[37] J.-S. Kim, D. Gračanin, K. Matković, and F. Quek, "Finger walking in place (FWIP): A traveling technique in virtual environments." International Symposium on Smart Graphics pp. 58-69, 2008.

[38] R. Kopper, F. Bacim, and D. A. Bowman, "Rapid and accurate 3D selection by progressive refinement." IEEE Symposium on $3 D$ User Interfaces (3DUI) pp. 67-74, 2011.

[39] J. J. LaViola Jr, E. Kruijff, R. P. McMahan, D. Bowman, and I. P. Poupyrev, 3D user interfaces: theory and practice: Addison-Wesley Professional, 2017. 
[40] R. W. Lindeman, "Bimanual interaction, passive-haptic feedback, 3 D widget representation, and simulated surface constraints for interaction in immersive virtual environments," George Washington University, 1999.

[41] J. F. Lucas, "Design and evaluation of 3D multiple object selection techniques," Virginia Tech, 2005.

[42] M. J. McKeown, L. K. Hansen, and T. J. Sejnowsk, "Independent component analysis of functional MRI: what is signal and what is noise?," Current opinion in neurobiology, vol. 13, no. 5, pp. 620629, 2003.

[43] D. Medeiros, F. Carvalho, L. Teixeira, P. Braz, A. Raposo, and I. Santos, "Proposal and evaluation of a tablet-based tool for 3D virtual environments," SBC Journal on Interactive Systems, vol. 4, no. 2, pp. 31, 2013.

[44] D. Medeiros, L. Teixeira, F. Carvalho, I. Santos, and A. Raposo, "A tablet-based 3d interaction tool for virtual engineering environments." Proceedings of the 12th ACM SIGGRAPH International Conference on Virtual-Reality Continuum and Its Applications in Industry pp. 211-218, 2013.

[45] D. Mendes, D. Medeiros, M. Sousa, E. Cordeiro, A. Ferreira, and J. A. Jorge, "Design and evaluation of a novel out-of-reach selection technique for VR using iterative refinement," Computers \& Graphics, vol. 67, pp. 95-102, 2017.

[46] M. R. Mine, "Virtual environment interaction techniques," UNC Chapel Hill CS Dept, 1995.

[47] A. Mossel, and C. Koessler, "Large scale cut plane: an occlusion management technique for immersive dense 3D reconstructions." Proceedings of the 22nd ACM Conference on Virtual Reality Software and Technology pp. 201-210, 2016.

[48] A. Mossel, B. Venditti, and H. Kaufmann, "DrillSample: precise selection in dense handheld augmented reality environments." Proceedings of the Virtual Reality International Conference: Laval Virtual pp. 10, 2013.

[49] H. Obermaier, and K. I. Joy, "An Automated Approach for Slicing Plane Placement in Visual Data Analysis," IEEE transactions on visualization and computer graphics, vol. 21, no. 12, pp. 1403-1414, 2015.

[50] A. Olwal, and S. Feiner, "The flexible pointer: An interaction technique for selection in augmented and virtual reality." Proc. UIST'03 pp. 81-82, 2003.

[51] R. R. Peeters, P. Kornprobst, M. Nikolova, S. Sunaert, T. Vieville, G. Malandain, R. Deriche, O. Faugeras, M. Ng, and P. Van Hecke, "The use of super-resolution techniques to reduce slice thickness in functional MRI," International Journal of Imaging Systems and Technology, vol. 14, no. 3, pp. 131-138, 2004.

[52] I. Poupyrev, N. Tomokazu, and S. Weghorst, "Virtual Notepad: handwriting in immersive VR." Proceedings. IEEE 1998 Virtual Reality Annual International Symposium (Cat. No. 98CB36180) pp. 126-132, 1998.

[53] A. Prior, "On-the-fly voxelization for 6 degrees-of-freedom haptic virtual sculpting." Proceedings of the 2006 ACM international conference on Virtual reality continuum and its applications pp. 263-270, 2006.

[54] G. Reitmayr, and D. Schmalstieg, "Mobile collaborative augmented reality." Proceedings IEEE and ACM International Symposium on Augmented Reality pp. 114-123, 2001.

[55] W. Ribarsky, J. Bolter, A. O. den Bosch, and R. Van Teylingen, "Visualization and analysis using virtual reality," IEEE Computer Graphics and Applications, vol. 14, no. 1, pp. 10-12, 1994.

[56] D. A. W. Rosa, and H. H. Nagel, "Selection techniques for dense and occluded virtual 3d environments, supported by depth feedback: Double, bound and depth bubble cursors." XXIX International Conference of the Chilean Computer Science Society pp. 218-225, 2010.

[57] S. Schkolne, M. Pruett, and P. Schröder, "Surface drawing: creating organic 3D shapes with the hand and tangible tools." Proceedings of the SIGCHI conference on Human factors in computing systems pp. 261-268, 2001.

[58] D. Schmalstieg, L. M. Encarnação, and Z. Szalavári, "Using transparent props for interaction with the virtual table," SI3D, vol. 99, pp. 147-153, 1999.
[59] G. Shan, M. Xie, Y. Gao, and X. Chi, "Interactive visual exploration of halos in large-scale cosmology simulation," Journal of Visualization, vol. 17, no. 3, pp. 145-156, 2014.

[60] S. Sridhar, A. M. Feit, C. Theobalt, and A. Oulasvirta, "Investigating the dexterity of multi-finger input for mid-air text entry." Proceedings of the 33rd Annual ACM Conference on Human Factors in Computing Systems pp. 3643-3652, 2015.

[61] S. Strothoff, D. Valkov, and K. Hinrichs, "Triangle cursor: Interactions with objects above the tabletop." Proceedings of the ACM International Conference on Interactive Tabletops and Surfaces pp. 111-119, 2011.

[62] E. Sundén, I. Lundgren, and A. Ynnerman, "Hybrid Virtual Reality Touch Table: An immersive collaborative platform for public explanatory use of cultural objects and sites." 15th Eurographics Workshop on Graphics and Cultural Heritage, Graz, Austria, September 27-29, 2017 pp., 2017.

[63] Z. Szalavári, and M. Gervautz, "The personal interaction Panel-a Two-Handed interface for augmented reality." Computer graphics forum pp. C335-C346, 1997.

[64] K. Takahashi. "Pcx - Point Cloud Importer/Renderer for Unity," 2019; https://github.com/keijiro/Pcx.

[65] L. Vanacken, T. Grossman, and K. Coninx, "Multimodal selection techniques for dense and occluded 3D virtual environments," International Journal of Human-Computer Studies, vol. 67, no. 3, pp. 237-255, 2009.

[66] J. Wang, and R. Lindeman, "Coordinated 3D interaction in tabletand HMD-based hybrid virtual environments." Proceedings of the 2nd ACM symposium on Spatial user interaction pp. 70-79, 2014.

[67] J. Wang, and R. W. Lindeman, "Object impersonation: Towards effective interaction in tablet-and HMD-based hybrid virtual environments." IEEE Virtual Reality (VR) pp. 111-118, 2015.

[68] L. Yu, K. Efstathiou, P. Isenberg, and T. Isenberg, "CAST: Effective and efficient user interaction for context-aware selection in 3D particle clouds," IEEE transactions on visualization and computer graphics, vol. 22, no. 1, pp. 886-895, 2016.

[69] L. Yu, K. Efstathiou, P. Isenberg, and T. Isenberg, "Efficient structure-aware selection techniques for 3D point cloud visualizations with 2DOF input," IEEE Transactions on Visualization and Computer Graphics, vol. 18, no. 12, pp. 22452254, 2012. 